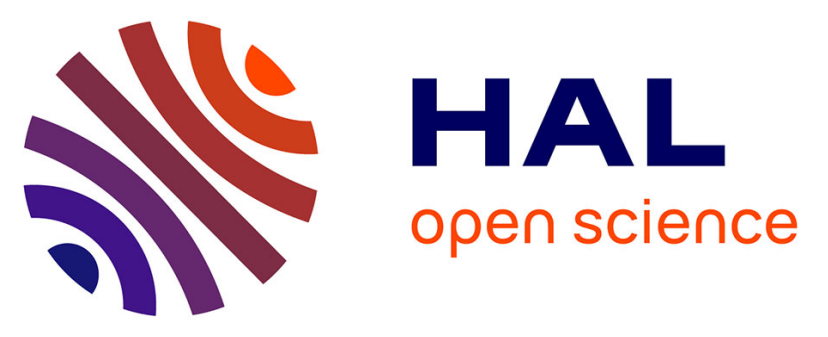

\title{
Polyethylene glycol Gold-Nanoparticles: Facile Nanostructuration of Doxorubicin and its complex with DNA molecules for SERS detection
}

Jolanda Spadavecchia, Ramesh Perumal, Sandra Casale, Jean-Marc Krafft, Christophe Methivier, Claire-Marie Pradier

\section{To cite this version:}

Jolanda Spadavecchia, Ramesh Perumal, Sandra Casale, Jean-Marc Krafft, Christophe Methivier, et al.. Polyethylene glycol Gold-Nanoparticles: Facile Nanostructuration of Doxorubicin and its complex with DNA molecules for SERS detection. Chemical Physics Letters, 2016, 648, pp.182-188. 10.1016/j.cplett.2015.08.038 . hal-01196383

\section{HAL Id: hal-01196383 \\ https: / hal.sorbonne-universite.fr/hal-01196383}

Submitted on 9 Sep 2015

HAL is a multi-disciplinary open access archive for the deposit and dissemination of scientific research documents, whether they are published or not. The documents may come from teaching and research institutions in France or abroad, or from public or private research centers.
L'archive ouverte pluridisciplinaire HAL, est destinée au dépôt et à la diffusion de documents scientifiques de niveau recherche, publiés ou non, émanant des établissements d'enseignement et de recherche français ou étrangers, des laboratoires publics ou privés. 


\title{
Polyethylene glycol Gold-Nanoparticles: Facile Nanostructuration of Doxorubicin and its complex with DNA molecules for SERS detection
}

\author{
Jolanda Spadavecchia ${ }^{a, b, c *}$, Ramesh Perumal ,Sandra Casale ${ }^{a, b}$, Jean-Marc \\ Krafft $^{\mathrm{a}, \mathrm{b}}$, Christophe Methivier ${ }^{\mathrm{a}, \mathrm{b}}$, Claire-Marie Pradier. \\ a Sorbonne Universités, UPMC Univ Paris 06,F-75005,Paris, France \\ ${ }^{\mathrm{b}}$ CNRS, UMR 7197,Laboratoire de Réactivité de Surface, Paris, France. \\ ${ }^{\circ}$ CNRS, UMR 7244, CSPBAT, Laboratoire de Chimie, Structures et Propriétés de \\ Biomatériaux et d'Agents Thérapeutiques Université Paris 13, Sorbonne Paris Cité, Bobigny, \\ France CNRS, Paris, France \\ *Corresponding author: jole23@libero.it

\begin{abstract}
We report the synthesis of dicarboxylic acid-terminated polyethylene-glycol (PEG)-gold nanoparticles by a simple one-step method, and their further use to form nanostructured surfaces for biomolecule immobilization. The synthesized nano-scale particles were conjugated with probe/target oligonucleotides in order to evaluate intercalation phenomenon in the presence of doxorubicin drug via Surface Enhanced Raman Spectroscopy (SERS) analysis.
\end{abstract}

KEYWORDS: PEG Gold Nanoparticles, Doxorubicin, Raman. 


\section{Introduction}

Gold nanoparticles (AuNPs) have attracted increasing attention due to their unique properties and interest in multiple research areas ${ }^{1,2}$ AuNPs exhibit different optical, electromagnetic and catalytic properties, from the bulk metal making them suitable for various nanotechnological applications ${ }^{2-4}$.

Recently, great advances have been made in the use of gold nanoparticles, for biomedical applications, owing to their stability, chemical reactivity, non-toxic nature and strong absorption and scattering properties ${ }^{5,6}$. For instance biomolecule- and/or biopolymerconjugated AuNPs are largely used as biomarkers or biodelivery vehicles, as well as for cosmetics, as anti-aging components for skin protection ${ }^{7,8}$. Therefore, more attention should be paid on efficient synthesis methods to match the enlarging demand of AuNPs. In the synthesis of nanoparticles a key role is played by the stabilizers, not only to avoid particle aggregation but also control their functional properties. To date, a variety of stabilizers have been employed for the synthesis of AuNPs ${ }^{9-10}$. Most of them are toxic, and the removal of excess stabilizer causes unwanted aggregation of the particles, which is a matter of concern for various clinical applications ${ }^{11}$.Biver et al ${ }^{12}$ synthesized Au nanoparticles using thioalkylated oligoethylene glycols functionalized with various fluorescent Acridine Orange derivatives ${ }^{13}$. Exchange of organic molecules on Au nanoparticles with PEG can inded be performed to prepare biocompatible PEG-stabilized $\mathrm{Au}$ nanoparticles ${ }^{5}$. Wang et al. synthesized a Polyethylene glycol (PEG)-modified gold nanoparticle complex by one-step reaction by synchrotron $x$-ray irradiation method ${ }^{14,15}$. A low concentration of unmodified PEG macromolecules is very important to control particle size and stabilize gold nanoparticles demonstrate high stability under realistic biomedical conditions ${ }^{16}$. Other approaches were applied to stabilize gold nanoparticles using sulfur-containing polymers, with a possible limitation of their suitability for specific biomedical application. As an example of biocompatible functionalization, polyethylene glycol (PEG) and poly N-vinyl-2-pyrrolidone (PVP) were exploited to modify nanovectors for gene drug/delivery ${ }^{17-19}$, tumor targeting ${ }^{20,21}$ 
and $x$-ray imaging ${ }^{22}$. For in vivo applications using PEG-gold nanoparticles, all the preparations were based on conventional methods (i.e.sodium citrate or sodium borohydrate reduction) and post-addition of thiolated PEG leading to gold particles usually larger than 20 $\mathrm{nm}^{23}$. Futhermore, the high cost of thiolated PEGs could limit commercial applications. And now as it is being synthesized with appropriate size scale and desired structures, it is getting much more enthusiasm such as biomedical applications particularly in targeted drug delivery therapy by one step approach. The multifunctional doxorubicin (DOX) conjugated AuNPs (DOX-AuNPs) may also be used to improve imaging contrast for photothermal cancer therapy ${ }^{24}$. Up to now DOX (figure 1) has been considered as an effective anticancer drug and its activity is apparently related to the direct interactions with dsDNA ${ }^{25,26}$, Therefore DOX is an inhibitor of the topoisomerase II and can inhibit DNA duplication and transcription to mRNA ${ }^{27}$. DOX-AuNPs facilitate intracellular drug delivery overcoming multidrug resistance in cancer cells ${ }^{28}$. The study of small ligands interacting with nucleic acids is a major area of research that has particular relevance in our understading of drug-DNA interactions involved in chemoterapeutic applications.From these studies it is known that drug binding can have many distinct modes including intercalation, and cross linking. We report here a one-solution synthesis to prepare polymer-modified gold nanoparticles using dycarboxylic PEG as stabilizer. We have thus implemented and evaluated a simple and reproducible method for labeling biomolecules with PEG gold nanoparticles without utilizing organic solvents and surfactants in order to improve the ability of drug molecules to reach specific cellular site and be easily released. The successive steps of functionalized PEG gold nanoparticle synthesis, as well as oligonucleotides and Doxorubicin grafting, have been characterized by Raman measurements. 


\section{Experimental Section}

\subsection{Materials}

Tetrachloroauric Acid $\left(\mathrm{HAuCl}_{4}\right)$, sodium borohydride $\left(\mathrm{NaBH}_{4}\right), \mathrm{N}$-hydroxysuccinimide (NHS), 1-(3-dimethylaminopropyl)-N'-ethylcarbodiimidehydrochloride $\quad$ (EDC),cysteamine (CYS), ethanol (Normapur 99\%), buffer solution (PBS pH: 7) ; Polyethylene glycol 600 Diacid (PEGdiacid), Doxorubicin hydrochloride ,Oligo DNA (NH2-AAC-CAT-AAT-TAA-AAA-TAC-CTCTAA-C), Target DNA (GTT-AGA-GGT-ATT-TTT-AAT-TAT-GGT-T), were purchased from Aldrich (Saint-Quentin Fallavier, France). All chemicals were used as such without further purification. Milli $Q$ water was used throughout the experiments.

2.2. Synthesis and bioconjugation of PEG-AuNPs: Li et al ${ }^{29}$ have reported a facile method to synthesize AuNPs from concentrated chloroauric acid by adding sodium hydroxide in the presence of citrate as stabilizer. We modified this protocol by adding dicarboxylic PEG as surfactant, in the mixture reaction ${ }^{30}$ (scheme 1). Briefly, $25 \mathrm{~mL}$ of Chloroauric acid $\left(\mathrm{HAuCl}_{4}\right)$ aqueous solution $\left(2.5 \times 10^{-4} \mathrm{M}\right)$ was added to $0.25 \mathrm{~mL}$ of Dicarboxylic PEG and mixed by magnetic stirring for $10 \mathrm{~min}$ at room temperature. To this solution, $20 \mathrm{~mL}$ of aqueous $0.01 \mathrm{M} \mathrm{NaBH}$ was added at once. The formation of the PEG-AuNPs was observed as an instantaneous color change of the solution from pale yellow to bright red after addition of the reducing agent. The as-prepared PEG-AuNPs solution was centrifugated at 15.000 rpm for 26 min for three times and then the supernatant was discarded and the residue was redispersed in an equivalent amount of buffer solution (PBS $\mathrm{pH}$ : 7). This was repeated twice principally to remove excess of dicarboxylic PEG. Stock solutions were stored at $27-29^{\circ} \mathrm{C}$ and characterized using UV-Vis spectroscopy and transmission electron microscopy (TEM).

\subsection{Characterization methods:}

The schematic diagram of the chemical immobilization method is depicted in scheme 2 $\underline{\text { SAMs formation: }}$

Self-assembling monolayers (SAMs) offer the possibility to modify the terminal functions of thiol-chains to bind any type of ligands by covalent, ionic or hydrogen interactions .Chemical procedures, based on SAMs of Cysteamine in absolute ethanol, have been described 
previously ${ }^{31}$. Briefly the freshly cleaned gold substrate was immersed in an unstirred $10 \mathrm{mM}$ ethanol solution of $\beta$-mercaptoethylamine (cysteamine) at room temperature, in the dark, for 6h. The gold substrate was then washed with ethanol and milli $Q$ water to remove the excess of thiol ( scheme 2a).

\section{PEG-AuNPs covalent grafting on Cysteamine Gold substrates:}

$2 \mathrm{ml}$ of PEG-AuNPs were dissolved in $2 \mathrm{ml}$ of buffer solution (PBS pH 7), in which $1 / 2$ eq of EDC/NHS was added, and stirred for $2 \mathrm{~h}$ (pre-activation). The colloidal solution was deposited on cysteamine-gold-coated surface for $12 \mathrm{~h}$ at room temperature ( scheme $2 \mathrm{~b}$ ).

Oligonucleotide linking to pegylated gold nanostructured surface:

The gold substrate, after PEG-AuNPs covalent grafting ${ }_{2}$ was treated with a solution of NHS (50 mg) and EDC (80 mg) in $3 \mathrm{~mL}$ of water for $4 \mathrm{~h}$ (activation) and then immersed successively in these solutions of Oligonucleotide in PBS buffer solution $(\mathrm{pH} 7)$. These experiments were carried out 4 times at room temperature leading to very similar results.

Probe Oligonucleotide (NH2-AAC-CAT-AAT-TAA-AAA-TAC-CTC-TAA-C) (100 microlitre /L in buffer solution) was deposited on the PEG-nano Au chip surface. After $1 \mathrm{~h}$, the surface was rinsed with buffer solution then with milli $\mathrm{Q}$ water for $1 \mathrm{~h}$. After this time, the surface was then washed with buffer, water and dried under a stream of nitrogen. The procedure was repeated two twice. Hybridization interaction was then evaluated by Oligonucleotide complementary target (GTT-AGA-GGT-ATT-TTT-AAT-TAT-GGT-T) during $1 \mathrm{~h}$. After this time, the surface was rinsed with buffer solution then with milli $Q$ water and dried by the same procedure ( scheme $2 \mathrm{c}$ ).

\section{Bioconjugation of Doxorubicin to Au-PEG Nanoparticles}

Bioconjugation of Doxorubicin to PEG-AuNPs (DOX-PEG-AuNPs) was described previously $^{31}$. Briefly, $20 \mu \mathrm{l}$ of an aqueous solution of EDC-NHS $(80 \mathrm{mg} / 20 \mathrm{mg})$ solution was added into $5 \mathrm{~mL}$ of PEG-AuNPs. After $2 \mathrm{~h}$, doxorubicin( $1 \mathrm{~mL}, 1 \mu \mathrm{M})$ was added and aged for $2 \mathrm{~h}$ and then centrifuged 3 times and redispersed in PBS solution to remove of unbound 
DOX. Scheme 2 e shows a schematic representation of the interaction between DOX-PEGAuNPs and Oligonucleotides onto gold nanostructured surface, compared to the DOX free interaction in the same conditions ( scheme $2 c$ ), in order to detect in a sensitive manner DNA hybridisation and intercalation events.

\section{Techniques}

\section{UV-Vis Absorption Spectroscopy.}

All the absorption spectra reported in this work have been recorded using a double-beam Varian Cary 500 UV-Vis spectrophotometer. In particular, absorption spectra of the PEGAuNPs were recorded in the $300-980 \mathrm{~nm}$ spectral range.

\section{SEM \& TEM}

SEM images were obtained using a SEM FEG Hitachi SU-70 scanning electron microscope with a low voltage of $1 \mathrm{kV}$, and distance of $1.5-2 \mathrm{~mm}$; the secondary electron detector "in Lens" was used. $50 \mu \mathrm{l}$ of colloidal solution was deposited onto a clean gold substrate and dried at room temperature for the SEM images.

Transmission electron microscopy measurements were recorded on a JEOL JEM 1011 microscope operating at an accelerating voltage of $100 \mathrm{KV}$. The TEM graphs were taken after separating the surfactant from the metal particles by centrifugation. Typically $1 \mathrm{~mL}$ of the sample was centrifuged for $20 \mathrm{~min}$ at a speed of $14000 \mathrm{rpm} / \mathrm{min}$. The upper part of the colourless solution was removed and the solid portion was re-dispersed in $1 \mathrm{ml}$ of water. $2 \mu \mathrm{L}$ of this re-dispersed particle suspension was placed on a carbon coated copper grid and dried at room temperature.

\section{SERS Spectroscopy:}

SERS Surface-enhanced Raman scattering spectra were recorded in the $500-1,900 \mathrm{~cm}-1$ range on a modular Raman spectrometer (Model HL5R of Kaiser Optical Systems, Inc.) equipped with a high-powered near-IR laser diode working at $785 \mathrm{~nm}$. Before spectra acquisition, an optical microscope (Olympus; objective, $\times 50$ ) was used to focus the laser beam. Measurements were carried using an objective $\times 100$. The laser output power was 10 
$\mathrm{mW}$, which corresponds to $\sim 1 \mathrm{~mW}$ on samples. For each spectrum, 30 acquisitions of $5 \mathrm{~s}$

were recorded to improve the signal-to-noise ratio. To ensure a representative characterization of surfaces, a minimum of three measurements were taken on different parts of the surface.

\section{Results}

\subsection{Synthesis and bioconjugation of PEG-AuNPs}

Figure.2 (left panel; black line) shows the adsorption band centered at $\approx 516 \mathrm{~nm}$ originating from the surface Plasmon of the gold nanoparticles ${ }^{32}$ with an average size of $7.2 \mathrm{~nm} \pm 0.5$ $\mathrm{nm}$. Thus indicating that gold nanoparticles were formed following the reduction of $\mathrm{AuCl}_{4}^{-33}$ The synthesis was carried out by reducing tetrachlororoauric acid $\left(\mathrm{HAuCl}_{4}\right)$ in the presence of PEG-diacid using sodium borohydride $\left(\mathrm{NaBH}_{4}\right)$ as a reducing agent. The color of the dispersion indeed instantly changed from yellow to red when sodium borohydride was added to a solution of gold precursor in the presence of PEG-diacid, confirming the formation of PEG-AuNPs in the solution. Figure.2 (right panel) reports the observed changes of the SPR band during hybridization between the DNA probes (dsDNA)(figure. 2-a),complementary DNA target sequences(ssDNA) (figure.2-b) and after intercalation of free DOX (figure.2-c) and DOX-PEG-AuNPs (figure.2-d). The spectrum (figure.2-a) displays a strong resonance peak at around $520 \mathrm{~nm}$ caused by SPR of individual nanoparticles after DNA immobilization. The progressive changes in the SPR band of the gold particles resulting from the hybridization with complementary target is apparent from the shift around $535 \mathrm{~nm}$ in the intensity of the SPR band towards higher wavelengths (figure.2-c). After interaction between free DOX , with DNA/DNA capped PEG-AuNPs, we observed a small peak at $535 \mathrm{~nm}$ and a second broadened band at $597 \mathrm{~nm}$. A dramatic shift at $640 \mathrm{~nm}$ was observed when DOX- 
PEG-AuNPs intercalate onto DNA oligonucleotides capped PEG-AuNPs (figure.2-c).These results clearly show that the shift in LSPR wavelength reflects small changes in the refractive index at the particle surface caused by the DNA/DNA, DNA/DNA/DOX, DNA/DNA DOXPEG-AuNPs events.

\subsection{Transmission electron microscopy (TEM) and Scanning electron microscopy (SEM) analysis of PEG-AuNPs}

A droplet (10 microL) of the colloidal solution, corresponding to the absorption spectra shown in figure. 2 (right panel black-line), was then deposited, on a microscope grid, leading to the transmission electron microscopy (TEM) image of figure.3 (a-b). In contrast to those reported in a very similar synthetic system, i.e.,CTAB/n-butanol/octane/water ${ }^{32}$, the TEM picture of PEG-AuNPs were well dispersed in size and shape. The histogram of 1623 particles fits a Gaussian behaviour with a mean size of $7.2 \mathrm{~nm}$ with a standard deviation of $0.5 \mathrm{~nm}$. Gold Nps were then covalently grafted on the cysteamine-modified gold surface. Surface organization and density were investigated using scanning electron microscopy (SEM). Figure.4 shows a typical SEM image of the planar gold surface after immobilization of PEGAuNPs on the cysteamine layer. A layer of well-defined, highly dispersed NPs on the gold surface has indeed been formed. The coverage of PEG-AuNPs onto gold surface was estimated by calculating the ratio between the surface area covered by gold nanoparticles and the total gold area ${ }^{32}$.The evaluation made from a of $1 \mathrm{x} 1 \mu \mathrm{m}^{2}$ image, leads to a surface NPs coverage, to $1.6 \times 10^{14}$ per square centimeter of the planar gold surface covered with Nps ,The height size distribution was made on the basis of 100 cross sections performed along the $1 \times 1 \mu \mathrm{m}^{2}$ image. The surface density is calculated as a ratio between the surface area covered by gold nanoparticles and the overall surface $\operatorname{area}^{36}$. Based of this equation, our surface density is $70 \%$. 


\subsection{SERS analysis of the interaction between DOX-PEG-AuNPs and oligonucleotides:}

Pegylated Gold Nanostructured surface were functionalized with amino probe oligonucleotides and our complementary target in order to evaluate the enhancement of Raman Signal before and after interaction of free DOX molecules and DOX-PEG-AuNPs. Stock solutions of doxorubicin, were prepared in phosphate-buffered saline (PBS at concentration of $10^{-3} \mathrm{M}$ and diluted to the $10^{-8} \mathrm{M}$ concentration before each experiment).

Experimental Raman (o SERS) spectra in the spectral range of $500-1900 \mathrm{~cm}^{-1}$ are shown in Figure 5. The spectral finger-print of $\mathrm{DOX}^{33}$ deposited onto pegylated gold nanostructured surface had several broad bands evident in figure 5 with the major peaks in the range 1100$1300 \mathrm{~cm}^{-1}$ and $1500-1600 \mathrm{~cm}^{-1}$ which correspond to the $\mathrm{C}=\mathrm{O}$ in plane deformation, $\mathrm{C}-\mathrm{O}-\mathrm{H}, \mathrm{C}-$ $\mathrm{H}$ skeletal ring vibrations, and hydrogen-bonded $\mathrm{C}=\mathrm{O}$ stretching modes, respectively. Figure 5-4 shows a SERS spectrum of double stranded DNA grafted onto pegylated gold nanostructured surface ( see scheme 2c). The intense peak at $731 \mathrm{~cm}^{-1}$ is assigned to the ring -breathing vibration of adenine ${ }^{33,34}$ and the weaker peak at $639 \mathrm{~cm}^{-1}$ corresponds to the ring-breathing mode of the other purine base, guanine. The localization of doxorubicin molecule in DNA, can influence the $\mathrm{NH}_{2}$ vibration of adenine molecule. Figure 5-3 shows the SERS spectra of $1,0 \times 10^{-8} \mathrm{M}$ of DOX after interaction with DNA. If we compare SERS spectra of free DOX onto pegylated gold nanostructured surface (figure 5-3), and DOX-DNA complex at the some conditions, reveal modification in the vibrational frequencies and intensities with disappereance of some bands upon complexation. The appareance of the $1591 \mathrm{~cm}^{-1}$ band confirm complex formation ${ }^{35,36,37}$. The intensity of the thymine band from at $1312 \mathrm{~cm}^{-1}$ increased because there is a deformation of the hydrogen bond between the $\mathrm{NH}_{2}$ group of adenine and the C40 group of thymine molecule. In the case of guanine, the band at 1467 $\mathrm{cm}^{-1}$ is caused by the $\mathrm{C} 8 \mathrm{H}-\mathrm{N} 9 \mathrm{C} 8$ and $\mathrm{C} 8 \mathrm{~N} 7$ guanine group vibrations ${ }^{38,39}$. The band at 1586 $\mathrm{cm}^{-1}$ (Ring breathing , Ring(Phe), $\mathrm{dC}-\mathrm{H}($ aro)) is very weak upon complexation. From this reason, we suggested that the interaction sites of doxorubicin were $\mathrm{C}-\mathrm{O}-\mathrm{NH}$ and ring phenil group. When DOX molecules were conjugated to the PEG AuNPs (see scheme 3 e), several RAMAN signal of DOX and the intercalation process in DNA molecules were significantly 
enhanced ${ }^{40}$. RAMAN spectrum was similar to that of free DOX molecules, except that all bands were broadened and the some new bands around $800-1100 \mathrm{~cm}^{-1}$ appeared. There is a blu-shift from $1372 \mathrm{~cm}^{-1}$ to $1312 \mathrm{~cm}^{-1}$ and a enhancement of the peak $1312 \mathrm{~cm}^{-1}$ due at the deformation of the hydrogen bond ,Ring $\mathrm{C} 20 / 15$ and $\mathrm{C} 16-\mathrm{OH}$ bending A prominent peak at $1123 \mathrm{~cm}^{-1}$ is due at C-N band and a small peak appears at $640 \mathrm{~cm}^{-1}$ may be due at C-S guanine $^{41}$. The SERS enanchement factor was calculated by comparing SERS signal excited of the DOX intercalated in DNA/DNA complex onto pegylated nanostructured surface before and after conjugation with PEG AuNPs. Here we adopted the estimation of the SERS enhancement factor reported by Gupta and Weimar ${ }^{42}$.Thus, the value of $\mathrm{G}$ was 0.47 .

\section{Discussion}

Particles formation and growth were controlled by the amphiphilic (dual nature) character of the PEG-diacid polymers described previously ${ }^{43,44}$. Other authors have reported the mechanism of gold nanoparticles formations in presence of poly (ethylene oxide)-poly (propylene oxide)-poly (ethylene oxide) block copolymers (PEO-PPO-PEO) varying temperature and solvent quality ${ }^{45}$.This block copolymers bind metal ions ${ }^{45}$, and reduction of bound $\mathrm{AuCl}_{4}^{-}$ions can proceed via oxidation of the oxyethylene and oxypropylene segments by the metal center ${ }^{45}$. The main difference with other synthetic procedures of PEG-AuNPs is that PEG-diacid is used in the same way as citrate for the stabilization of the particles through electrostatic interactions between the carboxylic acid groups and the gold surface ${ }^{44}$. Particle formation and growth were tuned by the amphiphilic character of the PEG-diacid polymer and include three steps: (1) reduction of $\mathrm{HAuCl}_{4}$ facilitated by dicarboxylic acidterminated PEG to form gold clusters; (2) adsorption of PEG diacid molecules on the surface of the gold clusters and reduction of metal ions in that vicinity; and (3) growth of gold particles and colloidal stabilization by PEG polymers. These findings indicate that PEGAuNPs, when immobilized on functionalized gold surface, via covalent link, preserve their size and shape. The low aggregation level observed may be due to the repulsive interactions between PEG-chains present at the surface of NPs. Note that E.M.S. Azzam et al also 
realized the chemisorption of gold nanoparticles on a self assembled monolayer (SAM) (decanedithiol)-modified gold surface; they observed the formation of $\mathrm{Np}$ aggregates on the surface due at the presence of ion charges of citrate molecules as surfactants that prevent a good dispersion after immobilization ${ }^{46}$.

Other authors M.H.Tu et al have demonstrated that aggregation and density depend on the nanoparticle size, smaller nanoparticles corresponding to higher surface densities ${ }^{47}$. The reason for this is that an AuNPs is normally covered by negative ions of citrate when synthetized. When the AuNPs is bigger in size, a large number of negative citrate ions would surround a single particle. When they are immobilized on the surface of a coverslip, it would be harder for a bigger particle to achieve high surface density than for a smaller one. Lee et al have reported a similar effect and noted that, achieving highest surface density might not be necessarily conjugated with better sensitivity of the sensor ${ }^{48}$. In our case the absence of ion charges of citrate and the presence of PEG chains as surfactant, allows a better coverage after funzionalization on gold surface without cluster aggregates. The covalent conjugation of DOX onto PEG-AuNPs was achieved using carbodiimide-based conjugation of the carboxylic-terminated polymer and the amine function of DOX and characterized by PM-IRRAS and X-Ray photoelectron microscopy (XPS $)^{49}$.The formation of an amide bond is evidenced by the presence of broad band at 1675 and an additional one at $1536 \mathrm{~cm}^{-1}$ (amide II $)^{32}$. This amide bond may be responsible of a different steric arrangement of DOX onto PEG-Au NPs and consequently interaction with DNA molecules.

The biological activity of the DOX molecule has been attributed to the formation of complex between the cromophore and the base pairs of DNA. In order to evaluate the intercalation effect of DOX and DOX-PEG-AuNPs, Dna oligonucleotides was binding onto gold nanoparticles (PEG-AuNPs) and monitored by UV-VIS spectroscopy. A dramatic blue-shift after interaction of DOX (figure 2-c) and DOX-PEG-AuNPs (figure 2-d) with oligonucleotides capped onto PEG-AuNPs, confirm the formation of complex.Several researchers have been demonstrate, the electron transfer mechanism and stability of intercalation of antraquinones (doxorubicin) into suitable DNA double helices that involve dipolar interactions highly . 
The comparison between the SERS spectrum of free DOX and that of the DOX-DNA/DNA complex onto gold nanostructured surface, show a loss intensity together the disappearance of some bands upon complexation. The use of this technique as a probe for the study of the molecular interaction of DOX (figure.1) and its complexes with DNA may allow us to have additional information on the changes in the spectra of DOX and on its interaction with DNA. A new band at $1273 \mathrm{~cm}^{-1}$ correspond to the ring stretching mode coupled with $v(\mathrm{C}-\mathrm{O})$ of ring A which is not intercalated within DNA. The band at $1642 \mathrm{~cm}^{-1}$ (assigned to hydrogen bonding to the $\mathrm{C}=\mathrm{O}$ ). Loss intensity of the bands at 1214 and $1246 \mathrm{~cm}^{-1}$, assigned to the vibrations involving in the plane $\mathrm{C}-\mathrm{O}, \mathrm{C}-\mathrm{O}-\mathrm{H}$ and $\mathrm{C}-\mathrm{H}$ bending modes, is observed. This confirms the intercalation of rings $B$ and $C$ whthin the double helix. The intensity increase of band at $1318 \mathrm{~cm}^{-1}$ and the red shift from 1586 to $1571 \mathrm{~cm}^{-1}$ of adenine are caused mainly by the doxorubicin interaction with the N7 position of adenine, which is accessible for doxorubicinin the DNA structure. Bin Kang et all have analysed SERS signal of Doxorubicin linked to gold nanoparticles of $30 \mathrm{~nm}$ of diameter by thiol group in order to evaluate the release of the drug in the cells ${ }^{39}$. The presence of AuNPs surface influences SERS signal and some bands are broadened. Conjugation of DOX to the AuNPs surface increases the Raman signal by the near -field plasmon of the AuNPs. In our case, the surface of AuNPs was modified by dicarboxyilic polyethilenglycol (PEG) to improve the biostability and reduce non specific binding of protein. The tickness of about $10 \mathrm{~nm}$ of pegylated gold nanostructured surface modified with DNA molecules, and the presence of PEG-AuNPs loaded with DOX improve the intercalation process with a impressive enhancement of SERS signal in the region $1300-1600 \mathrm{~cm}^{-1}$ at low concentration of Doxorubicin $\left(10^{-8} \mathrm{M}\right)$ due at double gold nanostructuration. The prominent peak at $1312 \mathrm{~cm}^{-1}$ explain the steric arrangement of DOXPEG-Au NPs by C-C ring and CO-NH that is different by chemical funzionalization studied by others in which DOX was binding by different linker thiol group. The peak at $450 \mathrm{~cm}^{-1}$, correspond to the $\mathrm{C}=\mathrm{O}$ in plane deformation of $\mathrm{DOX}{ }^{50}$; the decrase of SERS intensity at 450 $\mathrm{cm}^{-1}$ from 1 to 3 (see inset graph figure 5) confirms the interaction with oligonucleotides and success of the analysis. 


\section{Conclusions}

In this work, uniform PEG-AuNPs with low size polydispersity can be synthetized to developed a pegylated nanosructured surface that show a good sensitivity. These plateforms were used to build up a biosensor and test its reactivity and selectivity for a model antigen/antibody or amino oligonucleotides with your complementary target. Double gold nanostructuration by Doxorubicin as chemotherapeutic intercalator agent showed a better enhancement of SERS spectrum. The observed spectral changes, as a result of the complex formation, lead us to the following conclusions: the interaction of doxorubicin with DNA in an aqueous solution was realized by the drug binding to adenine and guanine. Doxorubicin interacted with the N7 position of adenine and guanine via a hydrogen bond formation between the $\mathrm{N} 7$ position of purine bases and the hydroxyl group of doxorubicin. We demonstrated that doxorubicin-modifed gold nanoparticles are efficient for the amplifcation of the SERS signal associated with DNA hybridization events. The stability of the DOX- PEGAuNPs over a long time when compared to PEG-AuNPs modifed with DNA makes the concept new and an interesting alternative for SERS based DNA sensing in an an easy manner. This approach will be extended to the realization of pegylated gold nanostructured surfaces in which different longer chains and functional groups of PEG polymers will be utilized to conjugate many type of tumoral biomarker in order to monitor the release of DOX in cancer cells.

\section{References:}

(1) Hu, M.; Chen, J.; Li, Z.-Y.; Au, L.; Hartland, G. V.; Li, X.; Marquez, M.; Xia, Y. Chemical Society Reviews (2006), 35.

(2) Wei, G.; Wang, L.; Sun, L.; Song, Y.; Sun, Y.; Guo, C.; Yang, T.; Li, Z. The Journal of Physical Chemistry C (2007), 1976-1982.

(3) Cui, R.; Liu, C.; Shen, J.; Gao, D.; Zhu, J.-J.; Chen, H.-Y. Advanced Functional Materials (2008), 18, 2197-2204.

(4) Zheng, Y.; Xiao, M.; Jiang, S.; Ding, F.; Wang, J. Nanoscale (2013) 788-795.

(5) Manson, J.; Kumar, D.; Meenan, B.; Dixon, D. Gold Bulletin (2011) 44, 99-105.

(6) Tian, L.; Gandra, N.; Singamaneni, S. ACS Nan (2013), 4252-4260.

(7) Zhenhai, G.; Jianhui, J.; Ting, Z.; Daocheng, W. J. Nanomaterials, (2011), 1-8. 
(8) Harshala J Parab, J.-H. H., Tsung-Ching Lai,; Yi-Hua Jan, R.-S. L., , Jui-Ling Wang, Michael Hsiao,; Chung-Hsuan Chen, Y.-K. H., Din Ping Tsai,; Pang, S.-Y. C. a. J.-H. S. Nanotechnology (2011), 395706.

(9) Liao, H.-G.; Jiang, Y.-X.; Zhou, Z.-Y.; Chen, S.-P.; Sun, S.-G. Angewandte Chemie International Edition (2008), 9100-9103.

(10) Jana, N. R. Small (2005), 1, 875-882.

(11) Smith, C.A.; Simpson, C.A.; Ganghyeok, K.; C. J., Carter; D. L., Feldheim,ACS Nano, 7 (5) (2013) 3991.

(12) Biver, T.; Eltugral, N.; Pucci, A.; Ruggeri, G.; Schena, A.; Secco, F.; Venturini, M. Dalton Transactions (2011), 40, 4190-4199.

(13) Biver T1, E. N., Pucci A, Ruggeri G, Schena A, Secco F, Venturini M. Dalton Transactions (2011), 40, 190-199.

(14) Zhao, T.; Wu, H.; Yao, S. Q.; Xu, Q.-H.; Xu, G. Q. Langmuir, 26, 14937-14942.

(15) Chang-HaiWang, C.-J. L., Cheng-Liang Wang, Tzu-En Hua,; Judy M Obliosca, K. H. L., Y Hwu, Chung-Shi Yang,; Ru-Shi Liu, H.-M. L., Jung-Ho Je and G Margaritondo J. Phys. D: Appl. Phys.(2008), 41, 195301.

(16) J.Manson, D.Kumar, B.J.Meenan Gold Bulletin (2011),99-105.

(17) Gaucher G1, A. K., Wang J, Leroux JC. Biomacromolecules. (2009), 2, 408-416.

(18) Pippa, N.; Pispas, S.; Demetzos, C.; Sivolapenko, G. Journal of Nanoparticle Research C7 - 2062, 15, 1-13.

(19) Prabaharan, M.; Grailer, J. J.; Pilla, S.; Steeber, D. A.; Gong, S. Biomaterials (2009), 6065-6075.

(20) Muthiah, M.; Park, I.-K.; Cho, C.-S. Biotechnology Advances (2013),1224-1236.

(21) Tejamaya, M.; RÃmer, I.; Merrifield, R. C.; Lead, J. R. Environmental Science \& Technology (2012), 7011-7017.

(22) Seol, S. K.; Kim, D.; Jung, S.; Chang, W. S.; Kim, J. T. Journal of Nanomaterials, (2013), 6.

(23) Gao J1, H. X., Liu H, Zan F, Ren J. Langmuir(2012), 4464-4471.

(24) Mirza, A. Z.; Shamshad, H. European Journal of Medicinal Chemistry (2011), 18571860.

(25) D'Incalci, M.; Badri, N.; Galmarini, C. M.; Allavena, P. Br J Cancer (2014), 645-650.

(26) PÃrez-Arnaiz, C.; Busto, N.; Leal, J. M.; GarcÃa, B. a. The Journal of Physical Chemistry B (2014), 1288-1295.

(27) Collins I.,Weber A., Levens D. Molecular and Cellular Biology (2011), 8437-8451

(28) Y.J. C.J Gu., C.W.Man, W.T.Wong, S.H.Cheng. Nanomedicine. (2012),204-211

(29) Li, C.; Li, D.; Wan, G.; Xu, J.; Hou, W. Nanoscale Research Letters (2011), 440.

(30) Spadavecchia, J.; Perumal, R.; Barras, A.; Lyskawa, J.; Woisel, P.; Laure, W.; Pradier, C.-M.; Boukherroub, R.; Szunerits, S. Analyst (2014), 157-164.

(31) Spadavecchia, J., Moreau, J., Hottin, J., Canva, M. Sensors and Actuators BChemical (2009), 139-143.

(32) Della Gaspera, E.; Guglielmi, M.; Martucci, A.; Giancaterini, L.; Cantalini, C. Sensors and Actuators B: Chemical (2012), 54-63.

(33) G. Das, A. N., L. Coluccio, F. Gentile, P. Candeloro, G. Cojoc, C. Liberale, F. De Angelis and E. Di Fabrizio Microsc. Res. Tech. (2011), 991.

(34) Chul Jae Lee, J. S. K., Mak Soon Kim, Kwang Pill Lee, and Mu Sang Lee* Bull. Korean Chem. Soc. (2004), 1211-1216.

(35) Beljebbar, A.; Sockalingum, G. D.; Angiboust, J. F.; Manfait, M. Spectrochimica Acta Part A: Molecular and Biomolecular Spectroscopy (1995), 2083-2090.

(36) Strekal, N. G., A.;Gacho,G.;Maskeivich,A.;Maskeivich, S. J.Mol.Struct (2001), 183.

(37) Stephen P. A. Fodor, R. P. R., Thomas R. Hays, and Thomas G. Spiro* J. Am. Chem. SOC (1985), 1520-1529.

(38) Stanic vá, J. F., G.; Chinsky, L.; utiak, V.; Mi kovsk P. J. Mol. Struct. (1999), 129.

(39) Kang, B.; Afifi, M. M.; Austin, L. A.; El-Sayed, M. A. ACS Nano (2013),7420-7427.

(40) Park, G.; Seo, D.; Chung, I. S.; Song, H. Langmuir (2013), 13518-13526. 
(41) Takae, S.; Akiyama, Y.; Otsuka, H.; Nakamura, T.; Nagasaki, Y.; Kataoka, K. Biomacromolecules (2005), 818-824.

(42) Gupta, W.A.weimar Chem Phys Lett.(2003), 302.

(43) Sakai, T.; Alexandridis, P. Langmuir (2005) , 8019-8025.

(44) Sakai, T.; Alexandridis, P. The Journal of Physical Chemistry B (2005), 7766-7777.

(45) Sakai, T.; Alexandridis, P. Langmuir (2004), 8426-8430.

(46) Azzam, E. M. S.; Bashir, A.; Shekhah, O.; Alawady, A. R. E.; Birkner, A.; Grunwald, C.; WÃ, C. Thin Solid Films (2009), 518, 387-391.

(47) Tu, M. H.; Sun, T.; Grattan, K. T. V. Sensors and Actuators B: Chemical (2014), 4353.

(48) Lee, J. S. Gold Bull (2010), 189.

(49) Spadavecchia J. ,Movia D., Moore C., Manus Maguire C., Moustaoui H., Casale S., Volkov Y., Prina-Mello A. Journal of Controlled release (2015)(submitted).

(50) Gu YJ, C. J., Man CW, Wong WT, Cheng SH. Nanomedicine.( 2012), 204-211. 


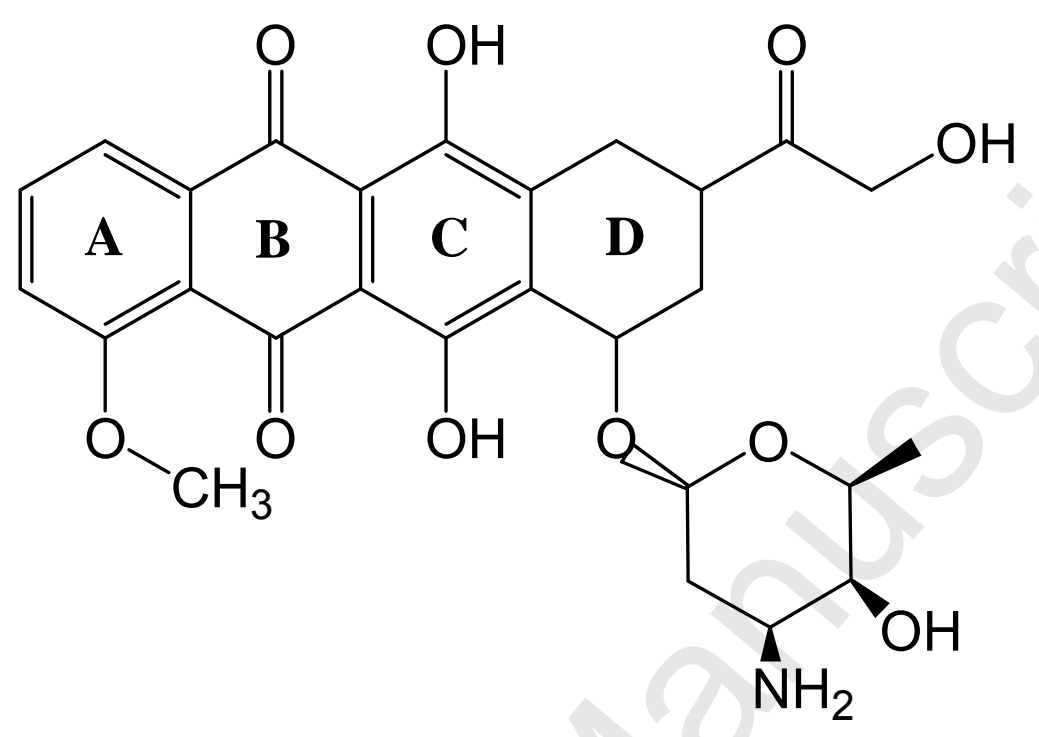

Figure.1 : Chemical structure of doxorubicin 

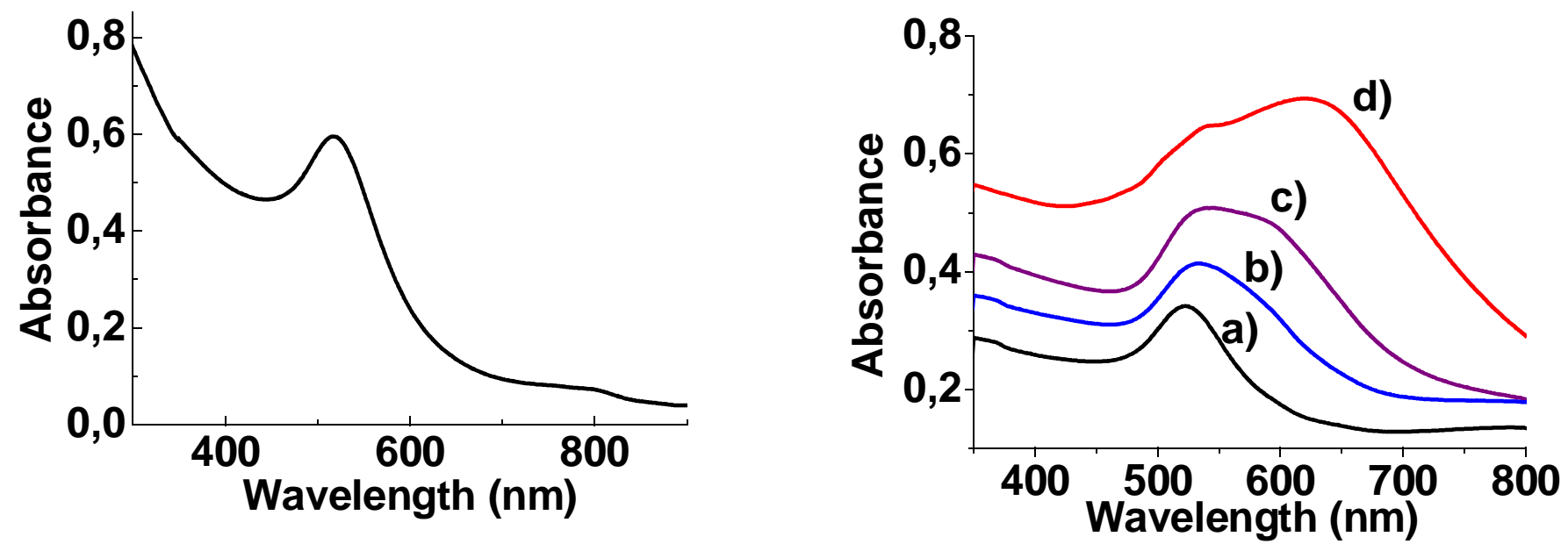

Figure.2 : UV-Vis spectra of the SPR band changes of PEG-AuNPs before (left panel $\lambda \max =516 \mathrm{~nm}$, black line) and after (right panel) (a) immobilisation dsDNA monolayer onto PEG-AuNPs (b) hybridization dsDNA/ssDNA monolayer onto PEGAuNPs (c) intercalation of doxorubicin free (DOX) onto PEG-AuNPs dsDNA/ssDNA oligonucleotides (d) intercalation of dox nanoparticles (DOX-PEG-AuNPs) onto PEG-AuNPs DNA oligonucleotides. 
(a)

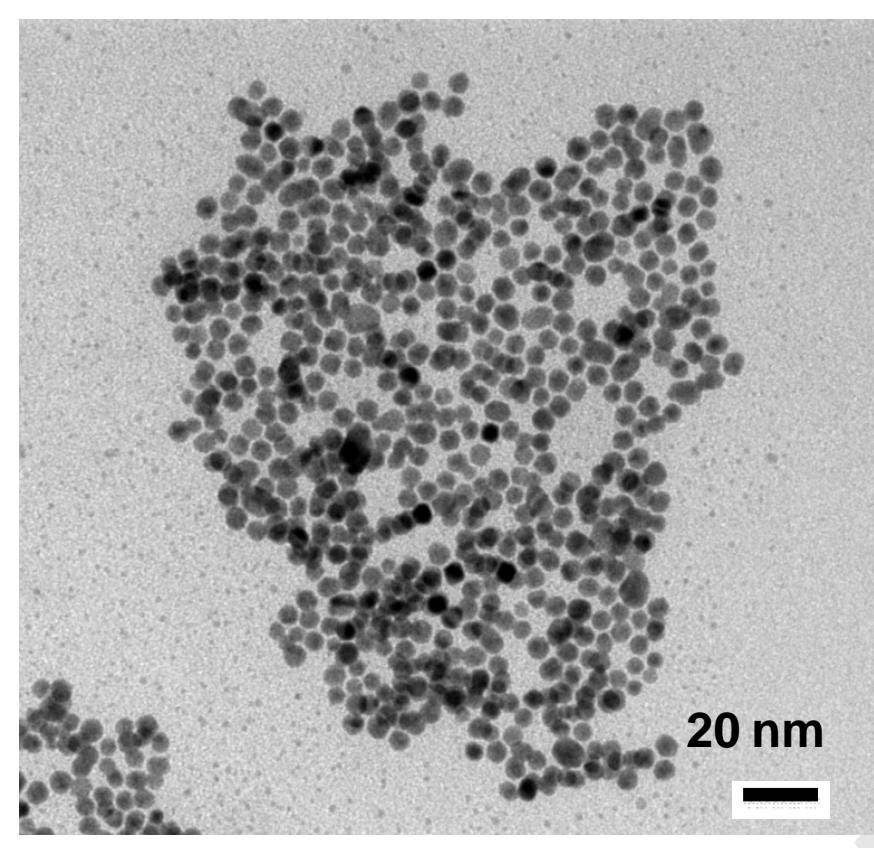

(b)

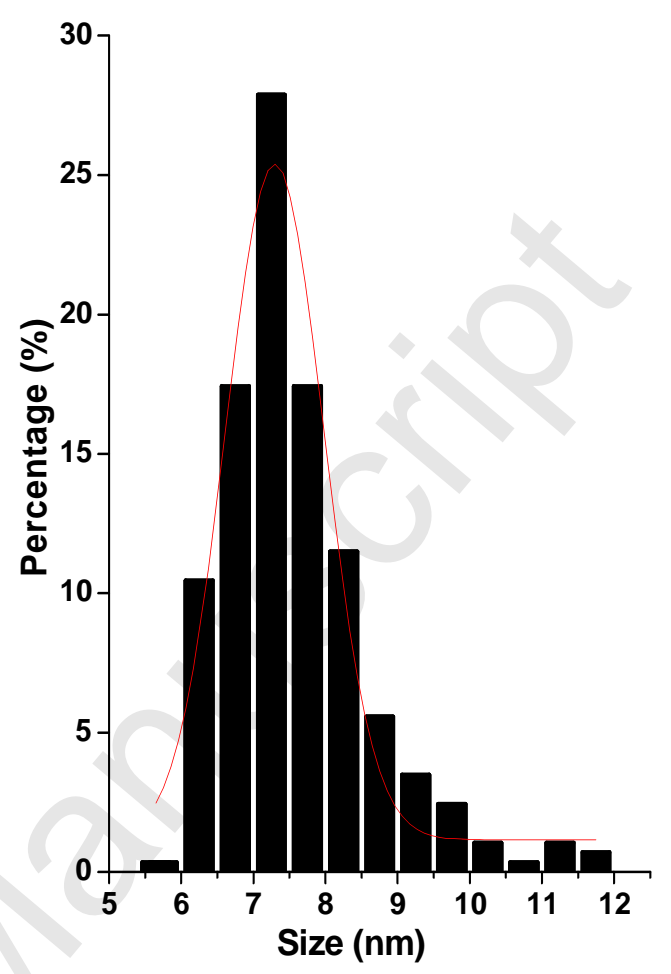

Figure. 3: Structural analysis of PEG-AuNPs: (a) TEM images, (b) particle size distribution 


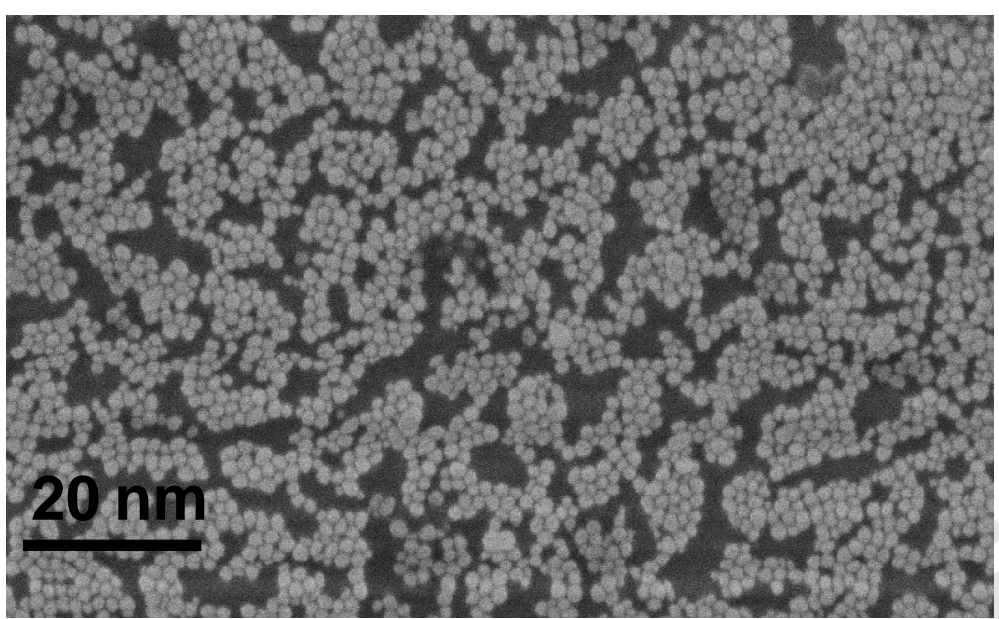

Figure. 4: SEM images of PEG-AuNPs immobilized on a planar gold surface

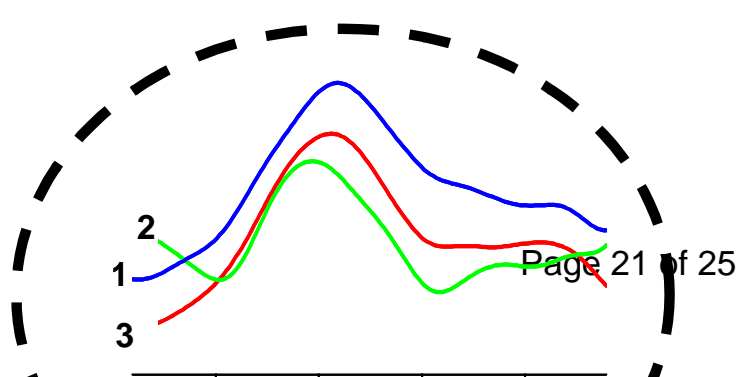




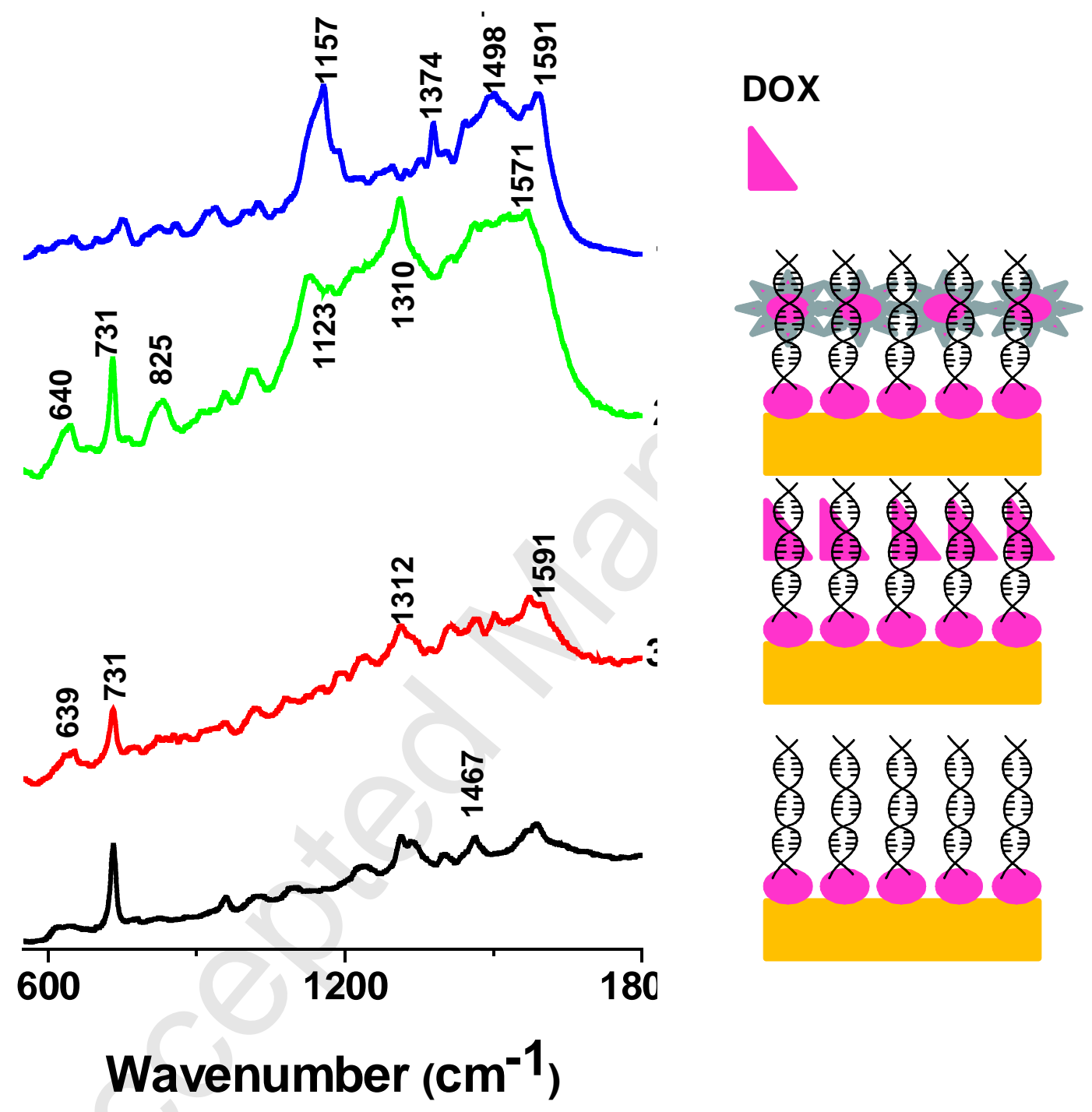

Figure. 5 Raman spectroscopy of DOX molecules free (1) and surface enhanced Raman spectra of DOX bound to PEG-AuNPs onto DNA-DNA pegylated gold nanostructured surface (2) Intercalation of DOX free onto DNA-DNA pegylated gold nanostructured surface (3) Compared DNA-DNA pegylated gold nanostructured surface onto CYS monolayer (4) (see inset graph) decrase of SERS intensity at 450 $\mathrm{cm}^{-1}$ 
<smiles>CC(C)OCCOCC(C)C(=O)O</smiles>
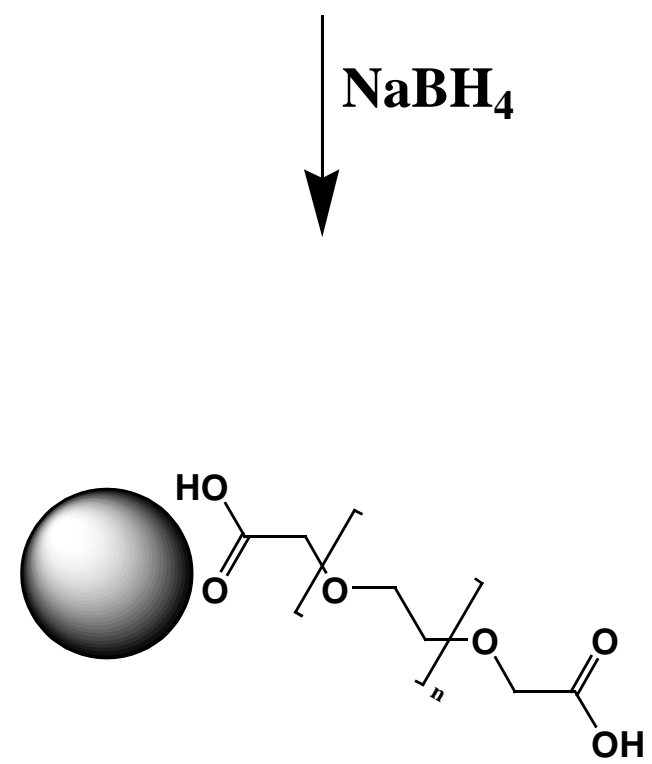

Scheme 1: Proposed formation mechanism of PEG-AuNPs in dicarboxylic acid-termninated PEG, $\mathrm{HAuCl}_{4}$ and $\mathrm{NaBH}_{4}$. 
(a)

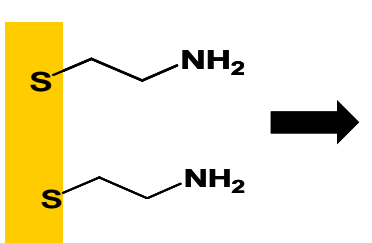

(b)

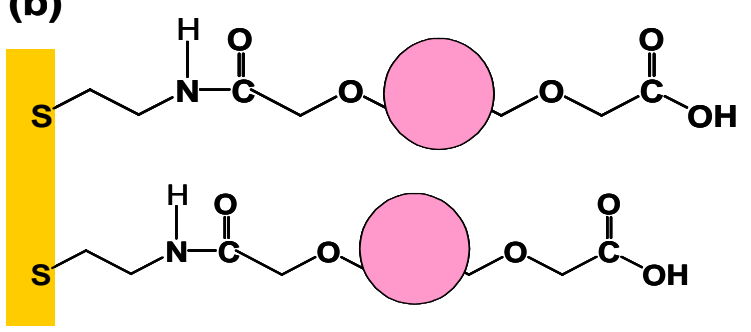

(c)

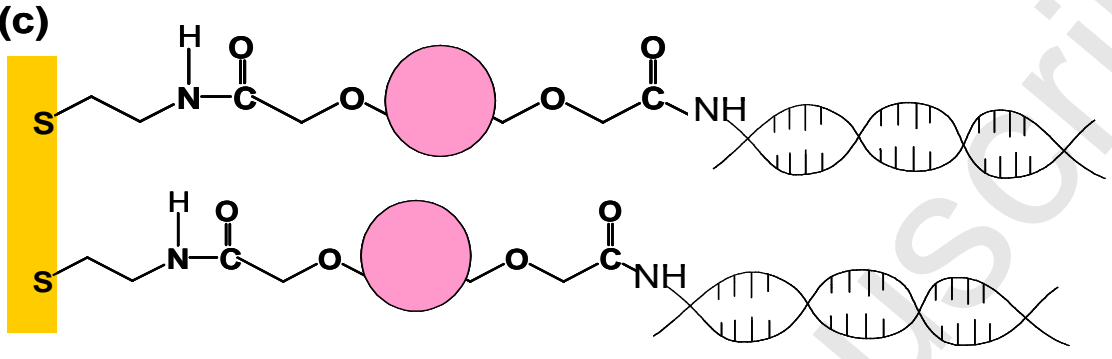

(d)
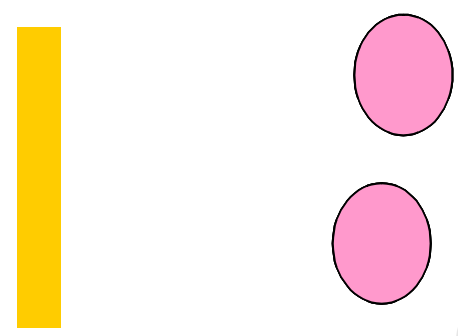

NH

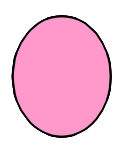

NH

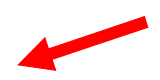

(e)

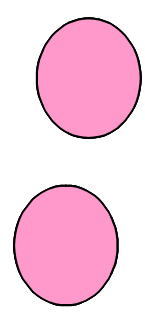

NH
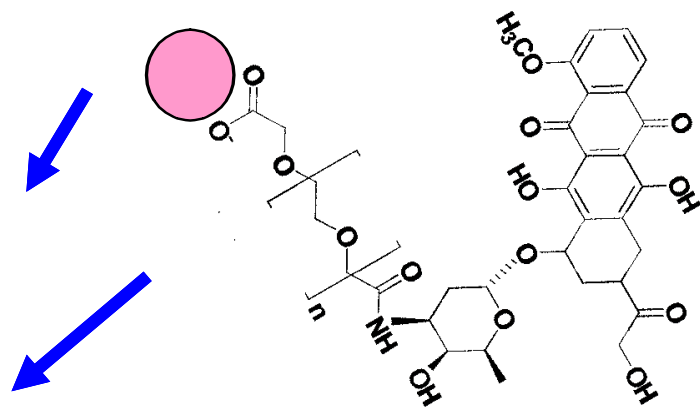

Scheme 2: Schematic representation of the biosensor elaboration strategy: (a) Au surface modification with cysteamine self-assembled monolayer, (b) PEG-AuNPs immobilisation via amide bond formed between activated $\mathrm{COOH}$ groups (using 
EDC/NHS) originating from NPs and amine group of cysteamine, (c) second activation of PEG-AuNPs and binding of amino oligonucleotide and complementary target recognition. (d) interaction of Doxorubicin with DNA onto Pegylated gold nanostructured surface. (e) interaction of Doxorubicin loaded to PEG-AuNPs with DNA onto Pegylated gold nanostructured surface. 